\section{Lecithin-Sphingomyelin-Ratio im Fruchtwasser}

\author{
A. M. Gressner ${ }^{1}$ und O. A. Gressner ${ }^{2}$ \\ ${ }^{1}$ Labor Dr. Wisplinghoff Berlin, Berlin, Deutschland \\ ${ }^{2}$ Labor Dr. Wisplinghoff Köln, Köln, Deutschland
}

Synonym(e) L/S-Ratio im Fruchtwasser

Englischer Begriff lecithin-sphingomyelin ratio in amniotic fluid

Definition Das Konzentrationsverhältnis von Lecithin zu Sphingomyelin im Fruchtwasser einer Schwangeren ist ein diagnostischer Indikator der fetalen Lungenreife.

Beschreibung Die Herabsetzung der Oberflächenspannung in den Lungenalveolen des Embryos ist für deren Entfaltung und somit für die Lungenreifung und -funktion von entscheidender funktioneller Bedeutung. Hierfür sind Surfaktantfaktoren ( $\triangleright$ Surfaktantfaktor) wie Glykoproteine, Lipide, Proteine, Lecithin und Sphingomyelin im Fruchwasser verantwortlich. Das Konzentrationsverhältnis von von der fetalen Lunge in das
Fruchtwasser sezernierte Lecithin (L) zu Sphingomyelin (S) gilt dabei als Kenngröße der Lungenreife. Bis zur 33. Schwangerschaftswoche (SSW) ist deren Konzentrationsverhältnis nahezu 1:1, danach steigt die Konzentration von Lecithin an, während die des Sphingomyelins nahezu unverändert bleibt. Ein L/S-Verhältnis von 2:1 oder höher weist auf Lungenreife hin, eine Ratio kleiner 1,5:1 auf Lungenunreife und wahrscheinliches Atemnotsyndrom. Ein Verhältnis zwischen 1,5 und 1,9:1 weist auf eine unreife Lunge und mögliche Atemprobleme hin. Das Probenmaterial ist die mit einer perkutanen Biopsie ( $\triangleright$ Amnionflüssigkeit) gewonnene, abzentrifugierte Amnionflüssigkeit. Alternativ ist eine Probengewinnung vaginaler Flüssigkeit möglich. Die Analytik wird mit der von L. Gluck entwickelten Methode der zweidimensionalen Dünnschichtchromatographie durchgeführt.

\section{Literatur}

Kulovich MV, Gluck L (1979) The lung profile I. Normal pregnancy. Am J Obstet Gynecol 135:57-63

Wijnberger LD, de Kleine M, Voorbij HA et al (2009) Prediction of fetal lung immaturity using gestational age, patient characteristics and fetal lung maturity tests. Arch Gynecol Obstet 281:15-21 